\title{
A CLASS OF DOMAIN FUNCTIONS AND SOME ALLIED EXTREMAL PROBLEMS
}

\author{
BY \\ ZEEV NEHARI
}

In this paper, we shall solve a number of extremal problems of which the following two are typical.

(a) Let $D$ denote a domain in the complex $z$-plane which is bounded by $n$ closed analytic curves $\Gamma_{\nu}\left(\nu=1, \cdots, n, \Gamma=\Gamma_{1}+\Gamma_{2}+\cdots+\Gamma_{n}\right)$, and let $\lambda\left(z_{0}\right) \quad\left(z_{0} \in \Gamma\right)$ be a positive function which is continuous on each $\Gamma_{\nu}$. Let further $B_{\lambda}$ denote the class of analytic functions $f(z)$ which are regular and single-valued in $D$, vanish at a given point $z=\zeta$ of $D$ and, for radial approach from within $D$, satisfy

$$
\limsup _{z \rightarrow z_{0}}|f(z)| \leqq \lambda\left(z_{0}\right), \quad z_{0} \in \Gamma
$$

Which is the precise upper bound for $\left|f^{\prime}(\zeta)\right|$ and for which function of $B_{\lambda}$ is it attained?

(b) Let $g(z)$ be regular and single-valued in $D$ and let $g(\zeta)=1$; if $\lambda\left(z_{0}\right)$ is defined as before, what is the precise lower bound for

$$
\int_{\Gamma} \lambda\left(z_{0}\right)\left|g\left(z_{0}\right)\right|^{2} d s, \quad d s=\left|d z_{0}\right|,
$$

and for which function $g(z)$ is it attained?

If $\lambda\left(z_{0}\right) \equiv 1$, problem (a) leads to a generalization of the classical Schwarz lemma to multiply-connected domains $[1,2,3]\left({ }^{1}\right)$ and its solution is found to be closely related to that of problem (b) $[2,4,5]$. The main result in this direction, due to P. R. Garabedian, from which all information relevant to the solution of problems (a) and (b) in this particular case can be deduced with little effort, is the following: there exist two functions $K(z, \zeta)$ and $L(z, \zeta)$ with the following properties. $K(z, \zeta)$ and $L(z, \zeta)+[2 \pi(z-\zeta)]^{-1}$ are regular in $D+\Gamma$, and $K(z, \zeta)$ and $L(z, \zeta)$ are connected by the identity $\left(^{2}\right)$

$$
[K(z, \zeta)]^{*} d s=\frac{1}{i} L(z, \zeta) d z, \quad z \in \Gamma,|d z|=d s .
$$

In our more general problem, involving an arbitrary positive and continuous boundary function $\lambda$, a similar central role is played by a pair of

Presented to the Society, February 26, 1949; received by the editors December 20, 1949.

(1) Numbers in brackets refer to the bibliography at the end of the paper.

(2) Complex conjugates are denoted by asterisks. 
domain functions $K_{\lambda}(z, \zeta)$ and $L_{\lambda}(z, \zeta)$. We shall first prove the existence of these functions and derive some of their main properties; once this is done, the solution of a number of extremal problems-including (a) and (b) -will be easily accomplished.

Theorem I. Let $D$ be a finite domain in the complex z-plane bounded by $n$ closed analytic curves $\Gamma_{\nu}\left(\nu=1, \cdots, n ; \Gamma_{1}+\Gamma_{2}+\cdots+\Gamma_{n}=\Gamma\right)$, and let $\lambda(z)$ $(z \in \Gamma)$ denote a positive function which is continous on each $\Gamma_{\nu}$. Then there exist two analytic functions $K_{\lambda}(z, \zeta)$ and $\dot{L}_{\lambda}(z, \zeta)$ with the following properties: $K_{\lambda}(z, \zeta)$ and $L_{\lambda}(z, \zeta)-[2 \pi(z-\zeta)]^{-1}$ are regular in $D ;\left|K_{\lambda}(z, \zeta)\right|$ is continuous in $D+\Gamma$ and $\left|L_{\lambda}(z, \zeta)\right|$ is continuous in $D+\Gamma-C_{\epsilon}$, where $C_{\epsilon}$ denotes a small open circle about $\zeta(\zeta \in D) ; K_{\lambda}(z, \zeta)$ and $L_{\lambda}(z, \zeta)$ are connected by the identity

$$
\lambda(z)\left[K_{\lambda}(z, \zeta)\right]^{*} d s=\frac{1}{i} L_{\lambda}(z, \zeta) d z, \quad z \in \Gamma,|d z|=d s .
$$

These properties determine both functions uniquely.

As a first step in the proof we consider the harmonic function

$$
u(z)=-\frac{1}{4 \pi} \int_{\Gamma} \log \lambda(t) \frac{\partial g(z, t)}{\partial n_{t}} d s_{t}, \quad d s_{t}=|d t|,
$$

where $g(z, t)$ is the Green's function of $D$, and the differentiation is performed with respect to the outwards pointing normal. Since $\lambda(t)$ is positive and continuous on each $\Gamma_{\nu}, \log \lambda(t)$ is also continuous there and the harmonic function $u(z)$ takes the boundary values $2^{-1} \log \lambda(t)$ on $\Gamma$. The harmonic conjugate $v(z)$ of $u(z)$ will generally not be single-valued in $D$; it will have periods about the boundary components $\Gamma_{\nu}$, which will be denoted by $2 \pi p_{\nu}, \nu=1, \cdots$, $n$. The function

$$
U(z)=u(z)+\sum_{\mu=1}^{n-1} \epsilon_{\mu} g\left(z, z_{\mu}\right), \quad z_{\mu} \in D, \mu=1, \cdots, n-1,
$$

where $\epsilon_{\mu}$ is either +1 or -1 , has the same boundary values as $u(z)$ since the Green's functions $g\left(z, z_{\mu}\right)$ vanish on $\Gamma$. Since the period of $g\left(z, z_{\mu}\right)$ about $\Gamma_{\nu}$ is $-(2 \pi)^{-1} \omega_{\nu}\left(z_{\mu}\right)$, where $\omega_{\nu}(z)$ denotes the harmonic measure of $\Gamma_{\nu}$ with respect to $D$-that is, the harmonic function in $D$ which takes the boundary values $\delta_{\nu \mu}$ on $\Gamma_{\nu}$-the period $2 \pi P_{\nu}$ of the harmonic conjugate $V(z)$ of $U(z)$ about $\Gamma_{\nu}$ will be given by

$$
P_{\nu}=p_{\nu}-\sum_{\mu=1}^{n-1} \epsilon_{\mu} \omega_{\nu}\left(z_{\mu}\right), \quad \quad \nu=1, \cdots, n .
$$

Our aim is to make the analytic function $\exp \{U(z)+i V(z)\}$ single-valued in $D$. This will obviously be achieved if we can determine the values $z_{\mu}$ and $\epsilon_{\mu}$ in such a way as to make the numbers $P_{\nu}$ in (4) equal to integers. It is clearly 
enough to do this for $\mathrm{n}-1$ of the $n$ periods $P_{v}$, since the sum of all $n$ periods must be equal to the sum of the periods due to the logarithmic poles, whence $P_{1}+\cdots+P_{n}=n-1$.

We therefore have to find $n-1$ points $z_{1}, \cdots, z_{n-1}$ in $D$ for which the equations

$$
\sum_{\mu=1}^{n-1} \epsilon_{\mu} \omega_{\nu}\left(z_{\mu}\right)=p_{\nu}+m_{\nu}, \quad \nu=1, \cdots, n-1,
$$

are satisfied for appropriately chosen integers $m_{\nu}$ and for a suitable choice of the $\epsilon_{\mu}$. The proof of the existence of $n-1$ points $z_{\mu}$ with these properties is equivalent to the solution of the "real part" of the classical Jacobi inversion problem on a closed Riemann surface of genus $n-1$ which, as shown by Schottky [6], can be obtained by suitably fitting together two replicas of $D$. However, we shall avoid reference to the rather complicated classical solution of the Jacobi inversion problem and shall instead give a simple direct proof for the existence of a solution of the system (5). That this is possible is due to the fact that the associated Riemann surface is symmetrical and that, therefore, our problem corresponds to a comparatively simple special case of the Jacobi problem.

We first remark that a schlicht conformal mapping of $D$ transforms the harmonic measures of $D$ into the harmonic measures of its conformal image; in order to solve the problem (5) for a whole conformal equivalence class, it is therefore sufficient to solve it for one particular domain of this class. As such a representative domain we shall choose the conformal image of $D$ by means of a mapping which transforms that part of the z-plane which is bounded by $\Gamma_{n}$ and contains $D$ into the upper half-plane. In order to avoid additional nomenclature, we shall retain the previous notations, thus denoting this new domain again by $D$, the corresponding complex variable by $z$, and so on. $\Gamma_{n}$ coincides with the real axis. Since the harmonic functions $\omega_{\nu}(z)(\nu=1, \cdots, n-1)$ vanish on the real axis, they can be analytically continued beyond the real axis by the identity $\omega_{\nu}\left(z^{*}\right)=-\omega_{\nu}(z)$. The functions $\omega_{\nu}(z)$ are thus harmonic in the entire domain $D^{\prime}$ which is obtained from $D$ by connecting with it, along the real axis, its mirror image with respect to that axis.

Since $\omega_{\nu}\left(z_{\mu}^{*}\right)=-\omega_{\nu}\left(z_{\mu}\right),(5)$ is equivalent to the system of equations

$$
\sum_{\mu=1}^{n-1} \omega_{\nu}\left(z_{\mu}\right)=p_{\nu}+m_{\nu}, \quad \nu=1, \cdots, n-1,
$$

if the $z_{\mu}$ are not restricted to points of $D$ but may coincide with any set of $n-1$ points of $D^{\prime}$.

Consider now the expression

$$
R=R\left(z_{1}, \cdots, z_{n-1}\right)=\sum_{\nu=1}^{n-1}\left[\sum_{\mu=1}^{n-1} \omega_{\nu}\left(z_{\mu}\right)-p_{\nu}-m_{\nu}\right]^{2} .
$$


$R\left(z_{1}, \cdots, z_{n-1}\right)$ is a continuous function of its variables in $D^{\prime}+\Gamma^{\prime}$, where $\Gamma^{\prime}$ denotes the boundary of $D^{\prime}$. Hence for a definite choice of integers $m_{\nu}, R$ has a non-negative minimum, which is attained for a set of $n-1$ points $z_{\mu}$ in $D^{\prime}+\Gamma^{\prime}$. We now choose the integers $m_{\nu}$ in such a way as to give to this minimum its smallest possible value; this is obviously possible since, in view of the fact that $-1 \leqq \omega_{\nu}(z) \leqq 1$, our choice is limited to a finite number of sets $\left\{m_{\nu}\right\}$. If $m_{1}, \cdots, m_{n-1}$ are the integers which give rise to the smallest minimum of $R$, we write $p_{\nu}+m_{\nu}=\gamma_{\nu}$, and we consider the solution of the problem

$$
R=R\left(z_{1}, \cdots, z_{n-1}\right)=\sum_{\nu=1}^{n-1}\left[\sum_{\mu=1}^{n-1} \omega_{\nu}\left(z_{\mu}\right)-\gamma_{\nu}\right]^{2}=\min ,
$$

where the $z_{\mu}$ may vary within $D^{\prime}+\Gamma^{\prime}$. As already mentioned, there exist minimizing sets $\left\{z_{\mu}\right\}$. It is easy to see that there must be at least one minimizing set which is entirely in $D^{\prime}$. Indeed, suppose $z_{1} \in \Gamma_{k}(k \neq n)$. Since $\omega_{k}\left(z_{1}\right)=1, \omega_{\nu}\left(z_{1}\right)=0(\nu \neq k)$, and $\omega_{\nu}(z)(\nu=1, \cdots, n-1)$ vanishes on the real axis, (7) will not be changed if $z_{1}$ is transferred to the real axis and, at the same time, $m_{k}$ is replaced by $m_{k-1}$. Similarly, a point $z_{\mu}$ may be transferred from the mirror image of $\Gamma_{k}$ to the real axis without altering the value of (7) if, at the same time, $m_{k}$ is replaced by $m_{k+1}$. Hence, there exists at least one minimizing set, say $S$, which is entirely in $D^{\prime}$. Moreover, this minimizing set can also be assumed not to contain both a point $z_{\mu}$ and its conjugate $z_{\mu}^{*}$; a glance at (8) shows that both $z_{\mu}$ and $z_{\mu}^{*}$ could be replaced by points on the real axis without altering the value of the minimum.

Since all points of $S$ are interior points of $D^{\prime}$, it follows from (8) that

$$
\frac{\partial R}{\partial z_{\mu}}=0, \quad \mu=1, \cdots, n-1, \quad \frac{\partial}{\partial z}=\frac{1}{2}\left(\frac{\partial}{\partial x}=i \frac{\partial}{\partial y}\right), \quad z=x+i y,
$$

at the points of $S$. We thus have the necessary conditions

$$
\sum_{\nu=1}^{n-1} a_{\nu} w_{\nu}^{\prime}\left(z_{\mu}\right)=0, \quad \quad \mu=1, \cdots, n \bullet 1,
$$

where

$$
a_{\nu}=\sum_{\mu=1}^{n-1} \omega_{\nu}\left(z_{\mu}\right)-\gamma_{\nu},
$$

and $w_{\nu}(z)$ is the analytic function whose real part is $\omega_{\nu}(z)$. Now there are two possibilities; either all the $a_{v}$ vanish, or else it follows from (9) that the derivative of the analytic function

$$
w(z)=\sum_{\nu=1}^{n-1} a_{\nu} w_{\nu}(z)
$$


vanishes at the $n-1$ points $z_{1}, \cdots, z_{n-1}$. In the first case, we have solved our problem, as a comparison of (10) and (6) shows. We thus have only to discuss the case in which at least one of the constants $a_{v}$ is different from 0 .

In this case, the points of $S$ are critical points of the function (11); if 2 or more of these points coincide, the critical points of (11) will be of corresponding multiplicity. Since the $a_{\nu}$ are real and, by the Schwarz symmetry principle, $w_{\nu}^{\prime}\left(z^{*}\right)=-\left[w_{v}^{\prime}(z)\right]^{*}$, the set $S^{*}$ of the points conjugate to those of $S$ is also a set of critical points of the function $w(z)$. Again, we have to distinguish between two cases, according as $S$ has points in common with the real axis, or not. If no point of $S$ is on the real axis, the sets $S$ and $S^{*}$ are disjoint, and we have thus located $2(n-1)$ critical points of $w(z)$. Obviously, $n-1$ of these points are interior to $D$. But this leads to a contradiction, since a function of the type (11) cannot have more than $n-2$ critical points in $D$, where each critical point is counted with its multiplicity; this is a well known property which can be easily proved by means of the argument principle and the fact that $i w^{\prime}(z) d z$ is real on $\Gamma$.

The only case yet to be discussed is therefore that in which one or more points of $S$ lie on the real axis while not all constants $a_{p}$ in (11) vanish. Again, this leads to a contradiction. Indeed, $R$ is constant on the real axis. If $z_{\mu}$ $=\operatorname{Re}\left\{z_{\mu}\right\}$ is a point of $S$, it may therefore be replaced by any other real point without altering the value of the minimum. Hence, all points of the real axis will be critical points of $w(z)$. In view of (11) this means that $\sum_{\nu=1}^{n-1} a_{\nu} w_{\nu}^{\prime}(z) \equiv 0$ on the real axis and, by analytic continuation, throughout $D^{\prime}$. But this is possible only if all the $a_{\nu}$ vanish, since the functions $w_{\nu}^{\prime}(z)$ $(\nu=1, \cdots, n-1)$ are linearly independent. The assumption that not all the $a_{\nu}$ vanish thus leads to a contradiction. This completes the proof of the possibility of solving the system (5).

As shown before, the function (3) which is constructed on the points $z_{\mu}$ solving (5) has a harmonic conjugate all of whose periods about the $\Gamma_{\nu}$ are integral multiples of $2 \pi$; the boundary values of $U(z)$ are $2^{-1} \log \lambda(t)$. If $V(z)$ denotes the harmonic conjugate of $U(z)$, we consider the analytic function

$$
q(z)=\exp \{U(z)+i V(z)\} .
$$

Since the periods of $U(z)+i V(z)$ are integral multiples of $2 \pi i, q(z)$ is free of periods about the $\Gamma_{\nu}$. The singularities of $U(z)$ in $D$-that is, as shown by (3), the points $z_{\mu}$-become simple zeros or simple poles of $q(z)$, depending on whether $\epsilon_{\mu}$ is equal to +1 or -1 . The analytic function $q(z)$ defined by (12) is therefore found to have the following properties: $q(z)$ is single-valued in $D$ and may have there zeros and poles whose combined number does not exceed $n-1$ (multiple zeros or poles are to be counted with their multiplicities); on the boundary, we have

$$
|q(z)|^{2}=\lambda(z), \quad z \in \Gamma
$$


As a next step in the construction of the functions $K_{\lambda}(z, \zeta)$ and $L_{\lambda}(z, \zeta)$ of Theorem I, we consider the functions

$$
\begin{gathered}
K^{(1)}(z, \zeta)=K(z, \zeta)+\sum_{\nu=1}^{\sigma} \alpha_{\nu} K\left(z, z_{\nu}\right)+\sum_{\nu=\sigma+1}^{n-1} \alpha_{\nu} L\left(z, z_{\nu}\right), \quad \sigma \leqq n-1, \\
L^{(1)}(z, \zeta)=L(z, \zeta)+\sum_{\nu=1}^{\sigma} \alpha_{\nu}^{*} L\left(z, z_{\nu}\right)+\sum_{\nu=\sigma+1}^{n-1} \alpha_{\nu}^{*} K\left(z, z_{\nu}\right)
\end{gathered}
$$

where $z_{1}, \cdots, z_{\sigma}(\sigma \leqq n-1)$ are the zeros of $q(z)$ and $z_{\sigma+1}, \cdots, z_{n-1}$ its poles, and $K(z, t)$ and $L(z, t)$ are the pair of functions connected by the identity (1) which were mentioned further above. The $n-1$ constants $\alpha_{\nu}$ shall be determined by the requirement that

$$
K^{(1)}\left(z_{\mu}, \zeta\right)=0, \mu=1, \cdots, \sigma ; L^{(1)}\left(z_{\mu}, \zeta\right)=0, \mu=\sigma+1, \cdots, n-1 .
$$

This will be possible if the system of $n-1$ linear equations

$$
\begin{gathered}
\sum_{\nu=1}^{\sigma} \alpha_{\nu} K\left(z_{\mu}, z_{\nu}\right)+\sum_{\nu=\sigma+1}^{n-1} \alpha_{\nu} L\left(z_{\mu}, z_{\nu}\right)=-K\left(z_{\mu}, \zeta\right), \quad \mu=1, \cdots, \sigma, \\
\sum_{\nu=1}^{\sigma} \alpha_{\nu}\left[L\left(z_{\mu}, z_{\nu}\right)\right]^{*}+\sum_{\nu=\sigma+1}^{n-1} \alpha_{\nu}\left[K\left(z_{\mu}, z_{\nu}\right)\right]^{*}=-\left[L\left(z_{\mu}, \zeta\right)\right]^{*}, \\
\mu=\sigma+1, \cdots, n-1,
\end{gathered}
$$

for the $n-1$ unknowns $\alpha_{1}, \cdots, \alpha_{n-1}$ has a solution. In order to show that this is the case, we first remark that we may assume that not all the numbers $K\left(z_{\mu}, \zeta\right)(\mu=1, \cdots, \sigma), L\left(z_{\mu}, \zeta\right)(\mu=\sigma+1, \cdots, n-1)$ are zero. If $q(z)$ has poles, this is certainly true, since $L(z, \zeta)$ has no zeros in $D[2]$; if $q(z)$ has no poles and all $K\left(z_{\mu}, \zeta\right)$ are zero, we may obviously identify $K^{(1)}(z, \zeta)$ and $L^{(1)}(z, \zeta)$ with $K(z, \zeta)$ and $L(z, \zeta)$, respectively. We have thus only to consider the case in which the system of linear equations is inhomogeneous. This system will have a solution if the associated homogeneous system can be shown not to possess a nontrivial solution; we proceed to show that such is indeed the case.

If the homogeneous system had a nontrivial solution, there would exist constants $\beta_{\nu}(\nu=1, \cdots, n-1)$, not all of which are zero, such that

$$
K^{(2)}\left(z_{\mu}\right)=0, \mu=1, \cdots, \sigma ; \quad L^{(2)}\left(z_{\mu}\right)=0, \mu=\sigma+1, \cdots, n-1,
$$

where

$$
\begin{array}{ll}
K^{(2)}(z)=\sum_{\nu=1}^{\sigma} \beta_{\nu} K\left(z, z_{\nu}\right)+\sum_{\nu=\sigma+1}^{n-1} \beta_{\nu} L\left(z, z_{\nu}\right), & \sigma \leqq n-1, \\
L^{(2)}(z) & =\sum_{\nu=1}^{\sigma} \beta_{\nu}^{*} L\left(z, z_{\nu}\right)+\sum_{\nu=\sigma+1}^{n-1} \beta_{\nu}^{*} K\left(z, z_{\nu}\right) .
\end{array}
$$


If two of the points $z_{\nu}$ coincide, at the point $z_{\tau}$, say $(\tau \leqq \sigma)$, then (14) and (14') will each have an extra term involving $\partial K\left(z, z_{\tau}\right) / \partial z_{\tau}^{*}$ and $\partial L\left(z, z_{\tau}\right) / \partial z_{\tau}$, respectively. The condition (15) which is lost by the two points coinciding is replaced by the condition $\partial K^{(1)}\left(z_{\tau}, \zeta\right) / \partial z_{\tau}=0$. If $\tau>\sigma$, the roles of the functions $K$ and $L$ in these definitions are interchanged. Coincidence of more than two points will result in the appearance of higher derivatives in the appropriate places. These changes do not affect the validity of the considerations which follow. The only property of the functions $K(z, \zeta)$ and $L(z, \zeta)$ which we use is the identity (1), and the same identity holds for the functions $\partial K(z, \zeta) / \partial \zeta^{*}$ and $\partial L(z, \zeta) / \partial \zeta$. Indeed, differentiating (1) with respect to $\operatorname{Re}\{\zeta\}$ and observing that $L(z, \zeta)$ is analytic in $\zeta$ while $K(z, \zeta)$ is analytic in $\zeta^{*}$, we obtain

$$
\left[\frac{\partial}{\partial \zeta^{*}} K(z, \zeta)\right]^{*} d s=\frac{1}{i} \frac{\partial}{\partial \zeta} L(z, \zeta) d z
$$

and similarly for higher derivatives.

In view of (1), we have

$$
\left[K^{(2)}(z)\right]^{*} d s=\frac{1}{i} L^{(2)}(z) d z, \quad z \in \Gamma,
$$

whence

$$
\begin{aligned}
& \frac{1}{i} K^{(2)}(z) L^{(2)}(z) d z \geqq 0, \\
& \frac{1}{i} \int_{\Gamma} K^{(2)}(z) L^{(2)}(z) d z>0 .
\end{aligned}
$$$$
z \in \Gamma
$$

Equality in (19) is excluded; this would mean that either $K^{(2)}(z)$ of $L^{(2)}(z)$ is identically zero, which is impossible since not all $\beta_{\nu}$ are zero. In view of Cauchy's theorem, (19) shows that the function $K^{(2)}(z) L^{(2)}(z)$ cannot be regular in $D$. This, however, leads to a contradiction, since the only singularities of $K^{(2)}(z)$ and $L^{(2)}(z)$ in $D$ are simple poles at the points $z_{\sigma+1}, \cdots, z_{n-1}$ and $z_{1}, \cdots, z_{\sigma}$, respectively, and these are cancelled by the zeros of $K^{(2)}(z)$ and $L^{(2)}(z)$ listed in (17). The assumption that the homogeneous system associated with (16) has a nontrivial solution thus leads to an absurdity; hence, the inhomogeneous system has a solution, which shows that the constants $\alpha_{1}, \cdots, \alpha_{n-1}$ in (14) and $\left(14^{\prime}\right)$ can indeed be so chosen as to make the functions $K^{(1)}(z, \zeta)$ and $L^{(1)}(z, \zeta)$ satisfy the conditions (15). We note that, in view of (1), (14), and $\left(14^{\prime}\right), K^{(1)}(z, \zeta)$ and $L^{(1)}(z, \zeta)$ are connected by the identity

$$
\left[K^{(1)}(z, \zeta)\right]^{*} d s=\frac{1}{i} L^{(1)}(z, \zeta) d z, \quad z \in \Gamma
$$


We now define the functions $K_{\lambda}(z, \zeta)$ and $L_{\lambda}(z, \zeta)$ as follows:

$$
\begin{aligned}
& K_{\lambda}(z, \zeta)=\frac{K^{(1)}(z, \zeta)}{q(z)[q(\zeta)]^{*}} \\
& L_{\lambda}(z, \zeta)=\frac{L^{(1)}(z, \zeta) q(z)}{q(\zeta)} .
\end{aligned}
$$

$K_{\lambda}(z, \zeta)$ is regular in $D$, since the zeros of $q(z)$ and the poles of $K^{(1)}(z, \zeta)$ are cancelled by the zeros of $K^{(1)}(z, \zeta)$ and the poles of $q(z)$, respectively; $L_{\lambda}(z, \zeta)$ is regular in $D$ except for its simple pole at $z=\zeta$, since all other poles of $q(z)$ and $L^{(1)}(z, \zeta)$ are cancelled by zeros of $L^{(1)}(z, \zeta)$ and $q(z)$, respectively. The residue of the simple pole of $L_{\lambda}(z, \zeta)$ at $z=\zeta$ is obviously equal to that of the pole of $L(z, \zeta)$ at this point, that is, to $(2 \pi)^{-1}$. [If $q(z)$ happens to have a zero or a pole at $z=\zeta$, the above definitions of $K_{\lambda}(z, \zeta)$ and $L_{\lambda}(z, \zeta)$ have obviously to be slightly modified. If $\eta$ is a point close to $\zeta$, we then define

$$
\left.K_{\lambda}(z, \zeta)=\lim _{\eta \rightarrow \zeta} K_{\lambda}(z, \eta), \quad L_{\lambda}(z, \zeta)=\lim _{\eta \rightarrow \zeta} L_{\lambda}(z, \eta)\right] .
$$

For $z \in \Gamma$, we have, by (20), (21), and (22),

$$
[q(z)]^{*} q(\zeta)\left[K_{\lambda}(z, \zeta)\right]^{*} d s=\frac{1}{i} \frac{L_{\lambda}(z, \zeta) q(\zeta)}{q(z)},
$$

whence

$$
|q(z)|^{2}\left[K_{\lambda}(z, \zeta)\right]^{*} d s=\frac{1}{i} L_{\lambda}(z, \zeta) d z, \quad z \in \Gamma .
$$

Comparison with (13) shows that $K_{\lambda}(z, \zeta)$ and $L_{\lambda}(z, \zeta)$ are indeed connected by the identity (2).

The continuity of $\left|K_{\lambda}(z, \zeta)\right|$ and $\left|L_{\lambda}(z, \zeta)\right|$ on $\Gamma$ can be shown as follows. By (2), we have

$$
\frac{1}{i} K_{\lambda}(z, \zeta) L_{\lambda}(z, \zeta) d z \geqq 0, \quad z \in \Gamma .
$$

Hence the analytic function $h(z)=\int_{z_{0}}^{z} K_{\lambda}(t, \zeta) L_{\lambda}(t, \zeta) d t, z_{0} \in \Gamma_{\nu}$, has a constant real part on each boundary component $\Gamma_{\nu}$; by the Schwarz inversion principle it is thus regular there (the $\Gamma_{\nu}$ are analytic curves). Thus the function $K_{\lambda}(z, \zeta) L_{\lambda}(z, \zeta)$ is regular on $\Gamma$. On the other hand, again by (2),

$$
\left|\frac{L_{\lambda}(z, \zeta)}{K_{\lambda}(z, \zeta)}\right|=\lambda(z)
$$

Since both the product and the ratio of $\left|K_{\lambda}\right|$ and $\left|L_{\lambda}\right|$ are continuous on $\Gamma$, this is also true for both functions separately. 
The only part of Theorem I yet to be proved is the unicity of the functions $K_{\lambda}$ and $L_{\lambda}$. Suppose there exists another pair of functions, say $K_{\lambda}^{\prime}(z, \zeta)$ and $L_{\lambda}{ }^{\prime}(z, \zeta)$, with the same properties. By $(2)$, we would have

$$
\left[K_{\lambda}(z, \zeta)-K_{\lambda}^{\prime}(z, \zeta)\right]^{*} d s=\frac{1}{i}\left[L_{\lambda}(z, \zeta)-L_{\lambda}^{\prime}(z, \zeta)\right] d z, \quad z \in \Gamma,
$$

where the function $L_{\lambda}-L_{\lambda}^{\prime}$ is regular in $D$, since the poles of $L_{\lambda}$ and $L_{\lambda}^{\prime}$, cancel each other. Multiplying both sides of $(24)$ by $K_{\lambda}(z, \zeta)-K_{\lambda}^{\prime}(z, \zeta)$ and integrating over $\Gamma$, we obtain, by Cauchy's theorem,

$$
\int_{\Gamma}\left|K_{\lambda}(z, \zeta)-K_{\lambda}^{\prime}(z, \zeta)\right|^{2} d s=0
$$

whence $K_{\lambda}(z, \zeta) \equiv K_{\lambda}^{\prime}(z, \zeta)$. The identity of $L_{\lambda}(z, \zeta)$ and $L_{\lambda}^{\prime}(z, \zeta)$ follows then from (24).

This completes the proof of Theorem I.

As a first application of Theorem I, we show that $K(z, \zeta)$ has the reproducing property

$$
\int_{\Gamma} \lambda(z) f(z)[K(z, \zeta)] * d s=f(\zeta)
$$

with respect to any function $f(z)$ which is regular and single-valued in $D$ and for which the integral $\int \lambda(z)|f(z)|^{2} d s$ exists in the Lebesgue sense. Indeed, by (2),

$$
\int_{\Gamma} \lambda(z) f(z)[K(z, \zeta)]^{*} d s=\frac{1}{i} \int f(z) L_{\lambda}(z, \zeta) d z,
$$

and this, by the residue theorem, is equal to $f(\zeta)$. We add that the functions $f(z)$ and $L_{\lambda}(z, \zeta)$ have radial limits almost everywhere on $\Gamma$, and that the residue theorem is valid with these radial limits defining these functions on $\Gamma$, since we may use a sequence of contours in $D$ approximating $\Gamma$ and apply the Lebesgue theorem to the resulting sequence of integrals.

Another property of $K_{\lambda}(z, \zeta)$ which follows immediately from (2) is its "hermitian" behavior if $z$ and $\zeta$ are interchanged, that is, the identity

$$
K_{\lambda}(\zeta, z)=\left[K_{\lambda}(z, \zeta)\right]^{*}
$$

From (2), we obtain

$$
\left[\frac{1}{i} K_{\lambda}(z, \zeta) L_{\lambda}(z, \eta) d z\right]^{*}=\frac{1}{i} K_{\lambda}(z, \eta) L_{\lambda}(z, \zeta) d z, \quad z \in \Gamma,
$$

where $\zeta$ and $\eta$ are two different points of $D$. By integration over $\Gamma$ and by the residue theorem, (26) follows. 
$L(z, \zeta)$ shows a different behavior if $z$ and $\zeta$ are interchanged. If the positive continuous function $\mu(z)$ is defined by

$$
\lambda(z) \mu(z)=1,
$$$$
z \in \Gamma
$$

it follows from (2) that

$$
\left[\frac{1}{i} K_{\lambda}(\eta, z) K_{\mu}(\eta, \zeta) d \eta\right]^{*}=\frac{1}{i} L_{\lambda}(\eta, z) L_{\mu}(\eta, \zeta) d \eta, \quad \eta \in \Gamma, z \in D .
$$

Integration over $\Gamma$ and the residue theorem yield $L_{\lambda}(\zeta, z)+L_{\mu}(z, \zeta)=0$, that is,

$$
L_{\lambda}(\zeta, z)=-L_{\mu}(z, \zeta),
$$

where $\lambda$ and $\mu$ are connected by (27). (28) shows, incidentally, that $L_{\lambda}(z, \zeta)$ is analytic in both variables.

We now show that the function $K_{\lambda}(z, \zeta)$ solves the extremal problem (b) stated at the beginning of this paper.

TheOREM II. Let $g(z)$ be regular and single-valued in $D$, let $\int_{\Gamma} \lambda(z)|g(z)|^{2} d s$ $<\infty$, and let $g(\zeta)=1$; then

$$
\int_{\Gamma} \lambda(z)|g(z)|^{2} d s \geqq \int_{\Gamma} \lambda(z)|M(z)|^{2} d s,
$$

where

$$
M(z)=\frac{K_{\lambda}(z, \zeta)}{K_{\lambda}(\zeta, \zeta)} .
$$

Equality in (29) is possible only for $g(z) \equiv M(z)$.

Proof. By (25),

$$
\int_{\Gamma} \lambda(z)\left|K_{\lambda}(z, \zeta)\right|^{2} d s=K_{\lambda}(\zeta, \zeta)
$$

and

$$
\int_{\Gamma} \lambda(z) g(z)\left[K_{\lambda}(z, \zeta)\right]^{*} d s=g(\zeta)
$$

Hence, by the Schwarz inequality,

$$
\begin{aligned}
1=|g(\zeta)|^{2} & \leqq \int_{\Gamma} \lambda(z)|g(z)|^{2} d s \int_{\Gamma} \lambda(z)\left|K_{\lambda}(z, \zeta)\right|^{2} d s \\
& =\left[K_{\lambda}(\zeta, \zeta)\right]^{-1} \int_{\Gamma} \lambda(z)|g(z)|^{2} d s,
\end{aligned}
$$


that is,

$$
\int_{\Gamma} \lambda(z)|g(z)|^{2} d s \geqq\left[K_{\lambda}(\zeta, \zeta)\right]^{-1}
$$

By (30) and (31),

$$
\int_{\Gamma} \lambda(z)|M(z)|^{2} d s=\left[K_{\lambda}(\zeta, \zeta)\right]^{-1}
$$

whence the theorem. In order to have equality in (29), it is plainly necessary that $g(z)\left[K_{\lambda}(z, \zeta)\right]^{*} \geqq 0$ and $|g(z)|=|M(z)|$ for $z \in \Gamma$; together with the fact that $g(\zeta)=M(\zeta)$, this means that $g(z)$ and $M(z)$ must be identical.

We next prove that $K_{\lambda}(z, \zeta)$ is a kernel function and can therefore be computed by means of a simple algorithm in terms of a complete set of functions.

Theorem III. Let $\mathcal{L}_{\lambda}^{2}$ denote the class of analytic functions $f(z)$ which are regular and single-valued in $D$ and for which $\int_{\Gamma} \lambda(z)|f(z)|^{2} d s<\infty$, where the integral is taken in the Lebesgue sense; let $u_{1}(z), u_{2}(z), \cdots$ be a set of functions in $\mathcal{K}_{\lambda}^{2}$ which is orthonormalized by the conditions

$$
\int_{\Gamma} \lambda(z)\left[u_{n}(z)\right]^{*} u_{m}(z) d s=\delta_{n m}
$$

and is complete in the sense that any function $f(z)$ in $\mathcal{L}_{\lambda}^{2}$ can be approximated by linear combinations $\sum_{\nu} a_{\nu} u_{\nu}(z)$ so that

$$
\int_{\Gamma} \lambda(z)\left|f(z)-\sum_{\nu} a_{\nu} u_{\nu}(z)\right|^{2} d s
$$

can be made arbitrarily small. Then

$$
K_{\lambda}(z, \zeta)=\sum_{\nu=1}^{\infty} u_{\nu}(z)\left[u_{\nu}(\zeta)\right]^{*},
$$

and this series converges absolutely and uniformly in every domain interior to $D$.

REMARK. Since the function $\lambda(z)$ is continuous, the class $\mathcal{L}_{\lambda}^{2}$ is, in fact, identical with the class of functions $f(z)$ for which $\int_{\Gamma}|f(z)|^{2} d s<\infty$. In the more general form used in the statement of the theorem, all considerations remain true for the case in which $\lambda(z)$ is an arbitrary positive function which is bounded away from 0 and $\infty$ and for functions $f(z)$ of the corresponding class $\mathcal{L}_{\lambda}^{2}$. Indeed, all results of this paper can be extended to these more general functions $\lambda(z)$. We shall, however, not do so, since this would require an excessive amount of real variable theory which would obscure the function-theoretic issues. 
Proof of Theorem III. Let $K_{n}(z, \zeta)$ be defined by

$$
K_{n}(z, \zeta)=\sum_{\nu=1}^{n} u_{\nu}(z)\left[u_{\nu}(\zeta)\right]^{*},
$$

where the functions $u_{\nu}(z)$ are in $\mathcal{L}_{\lambda}^{2}$ and are orthonormalized by (32). For any linear combination

$$
v_{n}(z)=\sum_{\nu=1}^{n} a_{\nu} u_{\nu}(z)
$$

we obviously have

$$
v_{n}(\zeta)=\int_{\Gamma} \lambda(z)\left[K_{n}(z, \zeta)\right] v_{n}(z) d s
$$

in particular,

$$
K_{n}(\zeta, \zeta)=\int_{\Gamma} \lambda(z)\left|K_{n}(z, \zeta)\right|^{2} d s
$$

The series

$$
\sum_{\nu=1}^{\infty}\left|u_{\nu}(\zeta)\right|^{2}=\lim _{n \rightarrow \infty} K_{n}(\zeta, \zeta)
$$

converges; indeed,

$$
\begin{aligned}
0 & \leqq \int_{\Gamma} \lambda(z)\left|K_{\lambda}(z, \zeta)-K_{n}(z, \zeta)\right|^{2} d s \\
& =K_{\lambda}(\zeta, \zeta)-K_{n}(\zeta, \zeta)+K_{n}(\zeta, \zeta)-K_{n}(\zeta, \zeta)=K_{\lambda}(\zeta, \zeta)-K_{n}(\zeta, \zeta),
\end{aligned}
$$

where (2) and (35) were used in the evaluation of the integral. Hence,

$$
K_{n}(\zeta, \zeta) \leqq K_{\lambda}(\zeta, \zeta)
$$

and the ascending sequence $\left\{K_{n}(\zeta, \zeta)\right\}$ converges. An immediate consequence of (38) is the absolute and uniform convergence of the series

$$
\sum_{\nu=1}^{\infty} u_{\nu}(z)\left[u_{\nu}(\zeta)\right]^{*}
$$

in every domain interior to $D$. Indeed,

$$
\left(\sum_{\nu=m}^{m+\sigma}\left|u_{\nu}(z)\right|\left|u_{\nu}(\zeta)\right|\right)^{2} \leqq \sum_{\nu=m}^{m+\sigma}\left|u_{\nu}(z)\right|^{2} \sum_{\nu=m}^{m+\sigma}\left|u_{\nu}(\zeta)\right|^{2}
$$

and this is arbitrarily small for sufficiently large $m$, since-in view of (38)the series (36) converges for every $\zeta \in D$. 
$K_{\lambda}(z, \zeta)$ is in $\mathcal{L}_{\lambda}^{2}$; for a given $\epsilon>0$, there exist therefore an integer $n_{0}$ and linear combinations $v_{n}(z)=\sum_{\nu=1}^{n} a_{\nu} u_{\nu}(z)$, such that, for $n>n_{0}$,

$$
\int_{\Gamma} \lambda(z)\left|K_{\lambda}(z, \zeta)-v_{n}(z)\right|^{2} d s \leqq \epsilon .
$$

Consider now the expression

$$
A=\int_{\Gamma} \lambda(z)\left[K_{n}(z, \zeta)\right]^{*}\left[K_{\lambda}(z, \zeta)-v_{n}(z)\right] d s .
$$

By (2) and (34), $A=K_{n}(\zeta, \zeta)-v_{n}(\zeta)$. By the Schwarz inequality,

$$
|A|^{2} \leqq \int_{\Gamma} \lambda(z)\left|K_{n}(z, \zeta)\right|^{2} d s \int_{\Gamma} \lambda(z)\left|K_{\lambda}(z, \zeta)-v_{n}(z)\right|^{2} d s .
$$

Hence, in view of (35), (38), and (39),

$$
\left|K_{n}(\zeta, \zeta)-v_{n}(\zeta)\right| \leqq \epsilon K_{\lambda}(\zeta, \zeta)
$$

whence

$$
\lim _{n \rightarrow \infty} K_{n}(\zeta, \zeta)=\lim _{n \rightarrow \infty} v_{n}(\zeta)
$$

On the other hand, again by (2), (39), and the Schwarz inequality,

$$
\begin{aligned}
\left|K_{\lambda}(\zeta, \zeta)-v_{n}(\zeta)\right|^{2} & =\left|\int_{\Gamma} \lambda(z)\left[K_{\lambda}(z, \zeta)\right]^{*}\left[K_{\lambda}(z, \zeta)-v_{n}(z)\right] d s\right|^{2} \\
& \leqq \int_{\Gamma} \lambda(z)\left|K_{\lambda}(z, \zeta)\right|^{2} d s \int_{\Gamma} \lambda(z)\left|K_{\lambda}(z, \zeta)-v_{n}(z)\right|^{2} d s \\
& \leqq \epsilon K_{\lambda}(\zeta, \zeta) .
\end{aligned}
$$

Hence $\lim _{n \rightarrow \infty} v_{n}(\zeta)=K_{\lambda}(\zeta, \zeta)$ and, in view of $(40)$,

$$
\lim _{n \rightarrow \infty} K_{n}(\zeta, \zeta)=K_{\lambda}(\zeta, \zeta)
$$

It therefore follows from (37) that

$$
\int_{\Gamma} \lambda(z)\left|K_{\lambda}(z, \zeta)-K_{n}(z, \zeta)\right|^{2} d s<\epsilon
$$

for sufficiently large $n$. Consider now the expression

$$
B=\int_{\Gamma} \lambda(z)\left[K_{\lambda}(z, \eta)-K_{n}(z, \eta)\right] *\left[K_{\lambda}(z, \zeta)-K_{n}(z, \zeta)\right] d s,
$$

where $\zeta$ and $\eta$ are points of $D$. By (2) and (35), we have 


$$
B=K_{\lambda}(\eta, \zeta)-K_{n}(\eta, \zeta)+K_{n}(\eta, \zeta)-\left[K_{n}(\zeta, \eta)\right]^{*}=K_{\lambda}(\eta, \zeta)-K_{n}(\eta, \zeta)
$$

On the other hand,

$$
|B|^{2} \leqq \int_{\Gamma} \lambda(z)\left|K_{\lambda}(z, \eta)-K_{n}(z, \eta)\right|^{2} d s \int_{\Gamma} \lambda(z)\left|K_{\lambda}(z, \zeta)-K_{n}(z, \zeta)\right|^{2} d s,
$$

whence, by (41),

$$
\left|K_{\lambda}(\eta, \zeta)-K_{n}(\eta, \zeta)\right|^{2} \leqq \epsilon^{2}
$$

for sufficiently large $n$. Therefore

$$
\lim _{n \rightarrow \infty} K_{n}(\eta, \zeta)=K_{\lambda}(\eta, \zeta)
$$

This completes the proof of Theorem III.

Our next theorem solves problem (a) stated at the beginning of this paper.

THEOREM IV. Let $B_{\lambda}$ denote the class of analytic functions which are singlevalued and regular in $D$, vanish at $z=\zeta(\zeta \in D)$ and, for radial approach from within $D$, satisfy

$$
\limsup _{z \rightarrow z_{0}}|f(z)| \leqq \lambda\left(z_{0}\right), \quad z_{0} \in \Gamma
$$

Then

$$
\left|f^{\prime}(\zeta)\right| \leqq F^{\prime}(\zeta)=2 \pi K_{\mu}(\zeta, \zeta)
$$

where

$$
\mu\left(z_{0}\right) \lambda\left(z_{0}\right)=1
$$

and

$$
F(z)=\frac{K_{\mu}(z, \zeta)}{L_{\mu}(z, \zeta)}
$$

If $L_{\mu}(z, \zeta) \neq 0$ for $z \in D, F(z)$ is in $B_{\lambda}$ and the inequality (43) is sharp; if $L_{\mu}(z, \zeta)$ (whose number of zeros in $D$ cannot exceed $n-1$ ) vanishes at the points $z_{1}, \cdots$, $z_{k}(k \leqq n-1)$, then (43) holds for the wider class of functions $f(z)$ which may have a simple or double pole at $z_{1}, \cdots, z_{k}$. For this wider class, (43) is sharp and equality holds again for the function (45).

Proof. In view of $(42), f(z)$ is bounded in $D$ and $\lim _{z \rightarrow z_{0}} f(z)$ exists therefore almost everywhere on $\Gamma$. With these boundary values, Cauchy's theorem yields

$$
f^{\prime}(\zeta)=\frac{2 \pi}{i} \int_{\Gamma} f(z) L_{\mu}^{2}(z, \zeta) d z
$$


whence, by (42),

$$
\left|f^{\prime}(\zeta)\right| \leqq 2 \pi \int_{\Gamma} \lambda(z)\left|L_{\mu}^{2}(z, \zeta) d z\right|
$$

and, by (2) and (44),

$$
\left|f^{\prime}(\zeta)\right| \leqq 2 \pi \int_{\Gamma}\left|K_{\mu}(z, \zeta) L_{\mu}(z, \zeta) d z\right|
$$

By (23),

$$
\frac{1}{i} K_{\mu}(z, \zeta) L_{\mu}(z, \zeta) d z \geqq 0 ;
$$

hence

$$
\left|f^{\prime}(\zeta)\right| \leqq \frac{2 \pi}{i} \int_{\Gamma} K_{\mu}(z, \zeta) L_{\mu}(z, \zeta) d z=2 \pi K_{\mu}(\zeta, \zeta),
$$

the last step following from Cauchy's theorem. Since the function $F(z)$ in (45) satisfies $2 \pi K(\zeta, \zeta)=F^{\prime}(\zeta)$, we have thus proved (43). In view of (2) and $(44)$,

$$
|F(z)|=\left|\frac{K_{\mu}(z, \zeta)}{L_{\mu}(z, \zeta)}\right|=\frac{1}{\mu(z)}=\lambda(z), \quad z \in \Gamma
$$

if $L_{\mu}(z, \zeta)$ is free of zeros in $D, F(z)$ is therefore in $B_{\lambda}$ and (43) is sharp. If $L_{\mu}(z, \zeta)$ has zeros, say at $z_{1}, \cdots, z_{k}$, then (45) and the ensuing argument evidently remain correct if $f(z)$ is allowed to have simple or double poles at $z_{1}, \cdots, z_{k}$. Equality in (43) will then again hold for the function $F(z)$ which now, in view of (45), will have simple poles at these points. Incidentally, the combined number of zeros of $K_{\mu}(z, \zeta)$ and $L_{\mu}(z, \zeta)$ in $D$ cannot exceed $n-1$; this is an immediate consequence of (23), the argument principle, and the fact that the total variation of arg $\{d z\}$ along $\Gamma$ is $-2 \pi(n-2)$.

If $\lambda(z) \equiv 1(z \in \Gamma)$, it can easily be shown that $L_{\lambda}(z, \zeta) \equiv L(z, \zeta)$ does not vanish in $D[2,4]$. If this were true in the case of a general $\lambda(z)$, the statement of Theorem IV would gain considerably in elegance. Unfortunately, however, this is not always the case, as can be shown by a simple counterexample. The function $K(z, \zeta)$ has $n-1$ zeros in $D[2,4]$. Let $\alpha$ be one of these zeros and consider the functions

$$
\begin{array}{r}
K_{0}(z, \zeta)=\frac{K(z, \zeta)}{(z-\alpha)\left(\zeta^{*}-\alpha^{*}\right)} \\
L_{0}(z, \zeta)=\left(\frac{z-\alpha}{\zeta-\alpha}\right) L(z, \zeta)
\end{array}
$$


By (1), we have

$$
\left[(z-\alpha) K_{0}(z, \zeta)\right]^{*} d s=\frac{L_{0}(z, \zeta)}{z-\alpha} d z
$$

whence

$$
|z-\alpha|^{2}\left[K_{0}(z, \zeta)\right]^{*} d s=\frac{1}{i} L_{0}(z, \zeta) d z .
$$

$K_{0}(z, \zeta)$ is regular in $D$ and $L_{0}(z, \zeta)$ has a simple pole of residue $(2 \pi)^{-1}$ at $z=\zeta$. From (2), (47), and the unicity property it follows therefore that $K_{0}(z, \zeta)$ and $L_{0}(z, \zeta)$ are identical with $K_{\lambda}(z, \zeta)$ and $L_{\lambda}(z, \zeta)$, respectively, for $\lambda(z) \equiv|z-\alpha|^{2}, z \in \Gamma$. But by its definition, the function $L_{0}(z, \zeta)$ has a zero at $z=\alpha$, which shows that functions $L_{\lambda}(z, \zeta)$ may indeed vanish in $D$. It would be interesting to find out what conditions have to be imposed on $\lambda(z)$ in order to exclude the existence of zeros of $L_{\lambda}(z, \zeta)$ in $D$.

We let follow an extremal problem which is solved by the function $L_{\lambda}(z, \zeta)$.

Theorem V. Let the function $h(z)$ be regular in $D$ except for a simple pole of residue $(2 \pi)^{-1}$ at $z=\zeta$; then

$$
\int_{\Gamma} \mu(z)|h(z)|^{2} d s \geqq \int_{\Gamma} \mu(z)\left|L_{\lambda}(z, \zeta)\right|^{2} d s,
$$

where

$$
\mu(z) \lambda(z)=1,
$$

Equality in (48) holds only for $h(z) \equiv L_{\lambda}(z, \zeta)$.

Proof. By Cauchy's theorem

$$
K_{\lambda}(\zeta, \zeta)=\frac{1}{i} \int_{\Gamma} h(z) K_{\lambda}(z, \zeta) d z .
$$

Hence, by the Schwarz inequality and (49),

$$
\begin{aligned}
K_{\lambda}^{2}(\zeta, \zeta) & \leqq \int_{\Gamma} \mu(z)|h(z)|^{2} d s \int_{\Gamma} \lambda(z)\left|K_{\lambda}(z, \zeta)\right|^{2} d s \\
& =K_{\lambda}(\zeta, \zeta) \int_{\Gamma} \mu(z)|h(z)|^{2} d s
\end{aligned}
$$

whence

$$
K_{\lambda}(\zeta, \zeta) \leqq \int_{\Gamma} \mu(z)|h(z)|^{2} d s
$$


Since, by (2) and (23),

$$
\int_{\Gamma} \mu(z)\left|L_{\lambda}(z, \zeta)\right|^{2} d s=\frac{1}{i} \int_{\Gamma} K_{\lambda}(z, \zeta) L_{\lambda}(z, \zeta) d z=K_{\lambda}(\zeta, \zeta),
$$

this proves (48). Retracing our steps, we find the equality can hold only if $-i h(z) K_{\lambda}(z, \zeta) d s \geqq 0$ and $|h(z)|=\left|L_{\lambda}(z, \zeta)\right|$ on $\Gamma$. In view of (23), this is possible only if $h(z)$ and $L_{\lambda}(z, \zeta)$ agree in both argument and absolute value for $z \in \Gamma$, which shows that they must be identical. This completes the proof.

By suitable specialization of the positive boundary function $\lambda$, a variety of other extremal problems can be solved. As an example, we consider the case in which

$$
\begin{aligned}
& \lambda(z)=\prod_{\nu=1}^{m}\left|z-\alpha_{\nu}\right|\left|\zeta-\alpha_{\nu}\right|^{-1}, \quad z \in \Gamma, \zeta \in D \\
& \mu(z)=\prod_{\nu=1}^{m}\left|\zeta-\alpha_{\nu}\right|\left|z-\alpha_{\nu}\right|^{-1}, \quad z \in \Gamma, \zeta \in D,
\end{aligned}
$$

where the $\alpha_{\nu}$ are arbitrary points of $D$. The following theorems hold:

THEOREM VI. Let $f(z)$ be regular and $|f(z)| \leqq 1$ in $D$, and let $f(\zeta)=f\left(\alpha_{1}\right)$ $=\cdots=f\left(\alpha_{m}\right)=0\left(\zeta, \alpha_{1}, \alpha_{2}, \cdots, \alpha_{m} \in D\right)$. Then

$$
\left|f^{\prime}(\zeta)\right| \leqq F^{\prime}(\zeta)=2 \pi K_{\lambda}(\zeta, \zeta)
$$

where $\lambda$ is defined in (50) and

$$
F(z)=\frac{K_{\lambda}(z, \zeta)}{L_{\lambda}(z, \zeta)} \prod_{\nu=1}^{m} \frac{\left(z-\alpha_{\nu}\right)}{\left(\zeta-\alpha_{\nu}\right)} .
$$

If $L_{\lambda}(z, \zeta) \neq 0$ in $D$, then $F(z)$ is bounded, and (52), is sharp. If $L_{\lambda}\left(z_{k}, \zeta\right)=0$ $\left(z_{k} \in D, k=1, \cdots, \sigma, \sigma \leqq n-1\right)$, then (52) holds, and is sharp, for the wider class of functions $f(z)$ which may have simple or double poles of $z=z_{k}$, and the extremal is again given by (53).

THEOREM VII. Let $g(z)$ be regular in $D$ apart from $m$ simple poles located at the points $\alpha_{1}, \cdots, \alpha_{m}$, let $g(\zeta)=0(\zeta \in D)$, and let $\lim \sup _{z \rightarrow \Gamma}|g(z)| \leqq 1$. Then

$$
\left|g^{\prime}(\zeta)\right| \leqq G^{\prime}(\zeta)=2 \pi K_{\mu}(\zeta, \zeta),
$$

where $\mu$ is defined in (51) and

$$
G(z)=\frac{K_{\mu}(z, \zeta)}{L_{\mu}(z, \zeta)} \prod_{\nu=1}^{m}\left(\frac{\zeta-\alpha_{\nu}}{z-\alpha_{\nu}}\right) .
$$

If $L_{\mu}(z, \zeta) \neq 0$ in $D$, then $G(z)$ satisfies the hypotheses of the theorem and (54) is sharp. If $L_{\lambda}(z, \zeta)$ has the zeros $z_{k}$ in $D$, then (54) is sharp for the wider class 
of functions $g(z)$ which may have simple or double poles at the points $z_{k}$. The extremal is also in this case given by (55).

Proof of Theorem VI. Since $f(\zeta)=f\left(\alpha_{1}\right)=\cdots=f\left(\alpha_{m}\right)=0$, we have, by the residue theorem,

$$
f^{\prime}(\zeta)=\frac{2 \pi}{i} \int_{\Gamma} f(z) \prod_{\nu=1}^{m}\left[\left(\zeta-\alpha_{\nu}\right)\left(z-\alpha_{\nu}\right)^{-1}\right] L_{\lambda}^{2}(z, \zeta) d z
$$

whence, in view of (51) and the boundedness of $f(z)$,

$$
\left|f^{\prime}(\zeta)\right| \leqq 2 \pi \int_{\Gamma} \mu(z)\left|L_{\lambda}^{2}(z, \zeta) d z\right|
$$

and, by virtue of (2), (23), and (50),

$$
\left|f^{\prime}(\zeta)\right| \leqq \frac{2 \pi}{i} \int_{\mathbf{r}} K_{\lambda}(z, \zeta) L_{\lambda}(z, \zeta) d z=2 \pi K_{\lambda}(\zeta, \zeta) .
$$

This proves (52). If $L_{\lambda}(z, \zeta) \neq 0$, the function $F(z)$ in (53) satisfies the hypotheses of the theorem. Indeed, $F(z)$ is regular in $D$ and vanishes at the required points. On $\Gamma$, we have, by (2), (50), and (53),

$$
|F(z)|=\frac{\lambda(z)\left|K_{\lambda}(z, \zeta)\right|}{\left|L_{\lambda}(z, \zeta)\right|} \equiv 1,
$$

which shows that $F(z)$ is bounded in $D$. If $L_{\lambda}(z, \zeta)$ vanishes at some points of $D$ (as shown above, the number of these points cannot exceed $n-1$ ), the extremal property of $F(z)$ within the class of functions having simple or double poles at these points is shown by modifying the argument in exactly the same way as in a similar case in the proof of Theorem IV.

The proof of Theorem VII is almost word for word the same as that of Theorem VI, the only difference being that the roles of $\lambda(z)$ and $\mu(z)$ are interchanged.

\section{BIBLIOGRAPHY}

1. Lars V. Ahlfors, Bounded analytic functions, Duke Math. J. vol. 14 (1947) pp. 1-11.

2. Paul R. Garabedian, Schwarz's lemma and the Szegö kernel function, Thesis, Harvard University, 1948; Trans. Amer. Math. Soc. vol. 67 (1949) pp. 1-35.

3. Zeev Nehari, Analytic functions possessing a positive real part, Duke Math. J. vol. 15 (1948) pp. 1033-1042.

4. - On bounded analytic functions, Proceedings of the American Mathematical Society vol. 1 (1950) pp. 268-275.

5. Menahem Schiffer, On various types of orthogonalization, Duke Math. J. vol. 17 (1950) (to appear).

6. F. Schottky, Über die konforme Abbildung mehrfach zusammenhängender ebener Flächen, J. Reine Angew. Math. vol. 83 (1887) pp. 300-351.

WASHINGTON UNIVERSITY,

ST. Louis, Mo. 